\title{
Erratum: Optical and phonon excitations of modified Pandey chains at the Si(111)-2 $\times 1$ surface [Phys. Rev. B 84, 155314 (2011)]
}

\author{
Charles H. Patterson ${ }^{0}$, Soumya Banerjee, and John F. McGilp
}

(Received 1 October 2019; published 15 October 2019)

DOI: 10.1103/PhysRevB.100.169904

The Si(111)- $(2 \times 1)$ modified Pandey $(\mathrm{MP})$ chain structures with positive $(+\mathrm{MP})$ and negative $(-\mathrm{MP})$ bucklings used in the original paper contained a stacking fault from the second Si bilayer downwards. Structures and optical and phonon excitation spectra obtained using the correct structure (shown in Fig. 1) are given here. Since structural parameters reported are surface atom bond lengths and excitations reported are strongly confined to the surface, changes in results due to the change in underlying bulk structure are mostly small. For example, the maximum change in reported surface bond lengths occurs for bonds 7-9 (0.013 $\AA)$ and 8-10 $(0.020 \AA)$ and $7^{\prime}-9^{\prime}(0.015 \AA)$ and $8^{\prime}-10^{\prime}(0.021 \AA)$ in the $+\mathrm{MP}$ and $-\mathrm{MP}$ structures. Atoms 9 and 10 and $9^{\prime}$ and $10^{\prime}$ were directly bonded to stacking fault $\mathrm{Si}$ atoms. Corrected bond lengths are reported in Table I. The difference in height of the Pandey chain $\mathrm{Si}$ atoms ( $b_{1}$ parameter) changes from 0.513 to $0.508 \AA$ in the + MP structure and from 0.559 to $0.561 \AA$ in the - MP structure. The total energy difference between the oppositely tilted structures was $4 \mathrm{meV}$ per surface unit cell in favor of the +MP structure. This value is unchanged in the corrected structure when a $6 \times 6$ MonkhorstPack $k$-space sampling grid is used. When a denser $8 \times 8$

TABLE I. Surface bond lengths in angstroms in MP structures from local density approximation (LDA) density functional theory (DFT), hybrid DFT, and low-energy electron diffraction (LEED).

\begin{tabular}{lccccc}
\hline \hline Atom pair & $-\mathrm{MP}^{\mathrm{a}}$ & $+\mathrm{MP}^{\mathrm{a}}$ & $-\mathrm{MP}^{\mathrm{b}}$ & $+\mathrm{MP}^{\mathrm{b}}$ & ${\text { LEED }+\mathrm{MP}^{\mathrm{c}}}^{2}$ \\
\hline 1 and 2 & 2.25 & 2.25 & 2.316 & 2.312 & 2.271 \\
1 -4 & 2.30 & 2.34 & 2.358 & 2.414 & 2.313 \\
2 and 3 & 2.34 & 2.30 & 2.411 & 2.352 & 2.347 \\
3 and 4 & 2.34 & 2.34 & 2.398 & 2.397 & 2.384 \\
4 and 5 & 2.41 & 2.37 & 2.470 & 2.427 & 2.371 \\
3-6 & 2.35 & 2.39 & 2.397 & 2.445 & 2.423 \\
5-7 & 2.34 & 2.33 & 2.372 & 2.382 & 2.359 \\
5-8 & 2.34 & 2.36 & 2.418 & 2.406 & 2.277 \\
6 and 7 & 2.33 & 2.34 & 2.349 & 2.363 & 2.393 \\
6-8 & 2.34 & 2.34 & 2.381 & 2.382 & 2.386 \\
7-9 & 2.30 & 2.30 & 2.331 & 2.330 & \\
8-10 & 2.40 & 2.39 & 2.443 & 2.445 & \\
Bulk & & & $2.371^{\mathrm{d}}$ & & 2.352 \\
\hline \hline
\end{tabular}

${ }^{a}$ LDA DFT Ref. [1].

${ }^{\mathrm{b}}$ Hybrid DFT (this Erratum, corrected).

${ }^{\mathrm{c}}$ Reference [2].

${ }^{\mathrm{d}}$ Hybrid DFT calculation on a bulk cell using the same basis set (this Erratum). grid is used for the corrected structure, the energy difference switches to $3 \mathrm{meV}$ in favor of the -MP structure.

Relative energies of surface states for the +MP and -MP structures are given in Table II and highest occupied molecular orbital and lowest unoccupied molecular orbital [(HOMO-LUMO) (HL)] and optical gaps from this Erratum, experiment, and several $G W$ calculations are given in Table III. The valence-band maximum (VBM) is at $\bar{K}$ for the $+\mathrm{MP}$ structure and at $\bar{J}$ for the $-\mathrm{MP}$ structure. The corrected HOMO-LUMO gaps are not significantly different from those

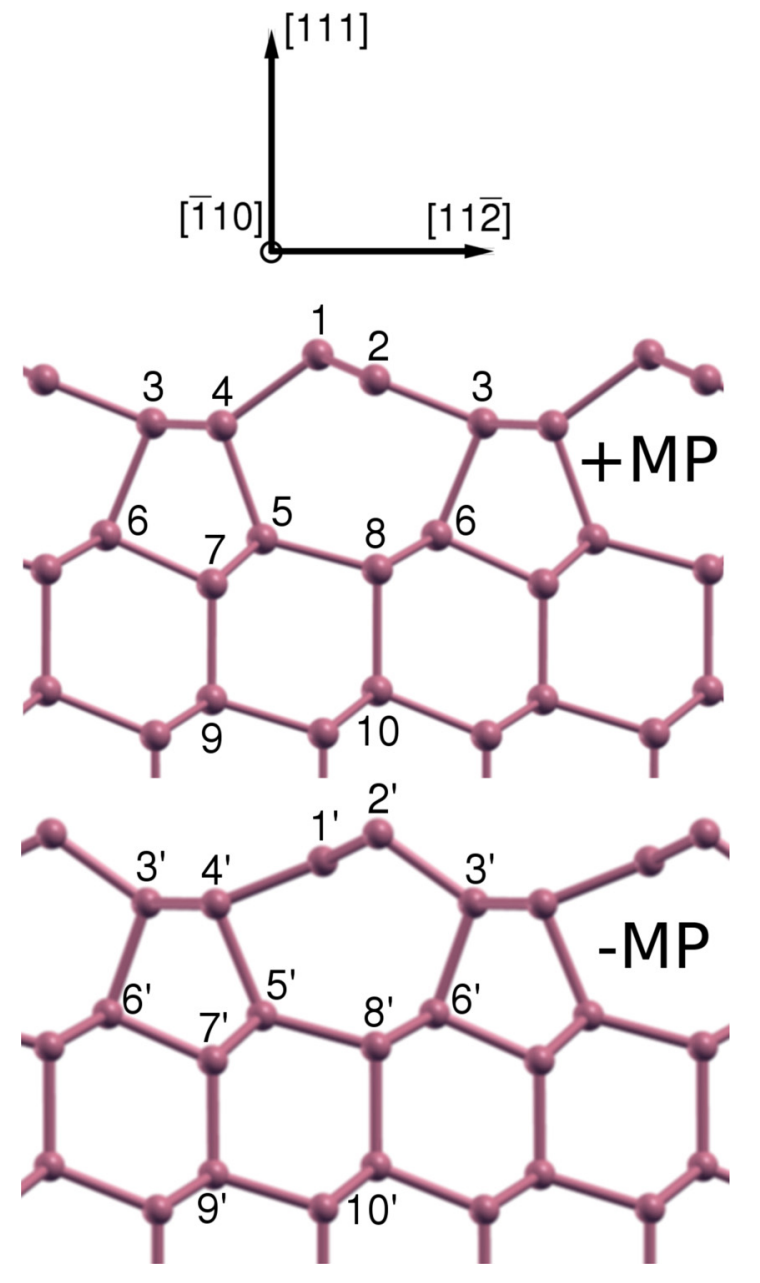

FIG. 1. MP chain model atomic structures with positive (+MP) and negative (-MP) tilts, viewed along the $[\overline{1} 10]$ (chain) direction. 
TABLE II. Positions of surface states in meV relative to the $\bar{\Gamma}$ point valence-band maximum in the + MP structure.

\begin{tabular}{lcc}
\hline \hline$k$ point & $+\mathrm{MP}$ & $-\mathrm{MP}$ \\
\hline $\bar{\Gamma}(\mathrm{VBM})$ & 0 & -5 \\
$\bar{J}$ & 40 & 131 \\
$\bar{K}$ & 46 & 117 \\
$\bar{J}$ (conduction band minimum) & 511 & 422 \\
\hline \hline
\end{tabular}

TABLE III. HL gap in \pm MP structures and optical excitation energies in the $+\mathrm{MP}$ structure in $\mathrm{eV}$ from scanning tunneling microscopy (STM) and angle-resolved photoemission spectroscopy (ARPES) experiment and hybrid DFT, $G W$, and Bethe-Salpeter equation calculations.

\begin{tabular}{lccc}
\hline \hline & HL gap + MP & HL gap -MP & Optical gap + MP \\
\hline STM $^{\mathrm{a}}$ & 0.83 & 0.47 & \\
ARPES $^{\mathrm{b}}$ & & 0.45 & \\
Hybrid DFT $^{\mathrm{c}}$ & 0.47 & 0.29 & 0.47 \\
$G W^{\mathrm{d}}$ & 0.75 & & 0.45 \\
$G W^{\mathrm{e}}$ & 0.62 & & 0.47 \\
$G W^{\mathrm{f}}$ & 0.69 & & 0.43 \\
$G W^{\mathrm{g}}$ & 0.79 & & \\
\hline
\end{tabular}

a STM data with levels referred to at the Fermi level, Ref. [3].

${ }^{\mathrm{b}}$ ARPES data for an $n$-type surface with both surface states occupied at the $\bar{J}$ point, Ref. [3].

${ }^{\mathrm{c}}$ This Erratum, corrected.

${ }^{\mathrm{d}}$ Calculation in a tight-binding basis using a fitted HL gap, Ref. [4].

${ }^{\text {e}}$ Plasmon pole approximation applied to diagonal elements of an inverse dielectric matrix, Ref. [5].

${ }^{\mathrm{f}}$ Generalized plasmon pole approximation, Ref. [6].

g DFT calculation with conduction bands shifted upwards by $0.5 \mathrm{eV}$ based on self-energy estimates at four $k$ points, Ref. [3].

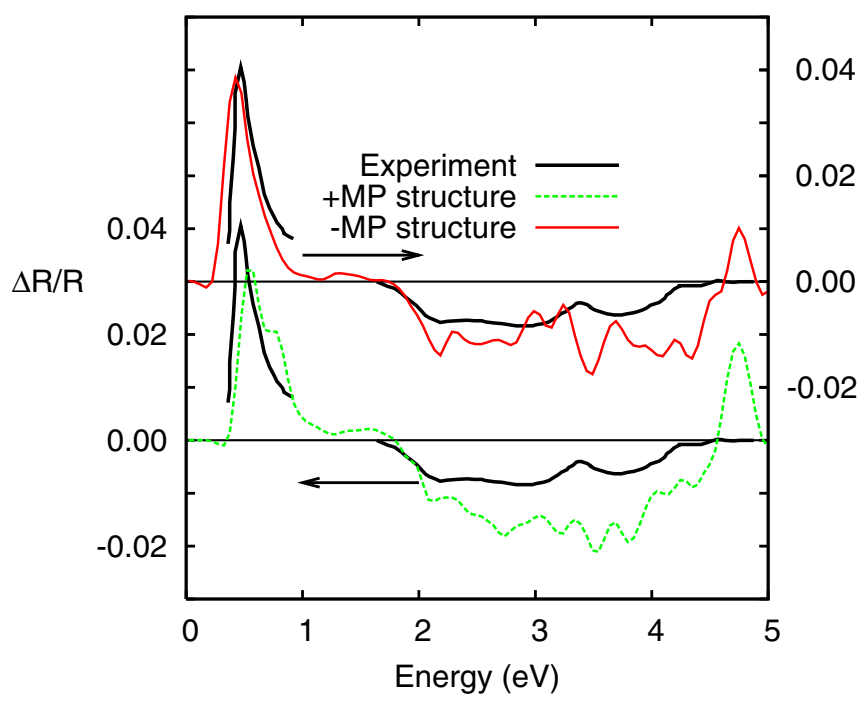

FIG. 2. Corrected reflectance anisotropy spectra for \pm MP structures compared to experimental data. Experimental data are redrawn from Ref. [7] and were collected in two spectral regions.
TABLE IV. Born effective charges in units of the electronic charge $e$ for surface atoms in the \pm MP structures.

\begin{tabular}{lcccc}
\hline \hline \multirow{2}{*}{+ MP structure } & & \multicolumn{2}{c}{-MP structure } \\
\cline { 5 - 6 } Atom & Born charge & & Atom & Born charge \\
\hline 1 & -2.74 & & $1^{\prime}$ & 3.83 \\
2 & 2.57 & & $2^{\prime}$ & -4.21 \\
3 & -0.08 & & $3^{\prime}$ & 0.09 \\
4 & 0.22 & & $4^{\prime}$ & -0.05 \\
5 & 0.07 & & $5^{\prime}$ & -0.24 \\
6 & -0.33 & & $6^{\prime}$ & 0.00 \\
7 & -0.06 & & $7^{\prime}$ & -0.04 \\
8 & 0.16 & & $8^{\prime}$ & 0.22 \\
\hline \hline
\end{tabular}

reported previously, however the highest occupied band at $\bar{\Gamma}$ is now lower than those at $\bar{J}$ and $\bar{K}$.

The reflectance anisotropy spectrum obtained with the corrected structure is shown in Fig. 2. Peak positions in the $+\mathrm{MP}$ and -MP reflectance anisotropy spectra are 0.55 and $0.44 \mathrm{eV}$ compared to 0.56 and $0.49 \mathrm{eV}$ previously. The experimental peak position is at $0.46 \mathrm{eV}$ [7].

Born effective charges for surface atoms are given in Table IV and mode energies and Born effective charges for surface active phonon modes are given in Table $\mathrm{V}$. The pattern of large and small atomic Born effective charges is the same for both corrected and uncorrected surface structures. The atom numbering scheme in Table IV is the same as in Fig. 1. The surface conductivity obtained from the phonon Born effective charges and mode energies in Table $\mathrm{V}$ is shown in Fig. 3. Overall, the conductivities of the corrected and uncorrected structures are similar. The +MP structure has a single strong mode polarized parallel to the Pandey chains at $51.0 \mathrm{meV}$ (previously $50.4 \mathrm{meV}$ ), and the -MP structure has

TABLE V. Mode energies (meV) and Born effective charges in phonon coordinates $Z_{p, i}$ in units of the electronic charge $e$ for phonons in the \pm MP structures.

\begin{tabular}{lrrr}
\hline \hline $\begin{array}{l}\text { Mode energy } \\
\text { +MP structure }\end{array}$ & \multicolumn{1}{c}{$Z_{x}$} & \multicolumn{1}{c}{$Z_{y}$} & \multicolumn{1}{c}{$Z_{z}$} \\
\hline 17.5 & 0.01 & -0.28 & 0.03 \\
20.7 & -0.31 & -0.03 & 0.03 \\
47.3 & 0.13 & 0.01 & 0.00 \\
51.0 & 0.00 & -1.85 & 0.00 \\
55.3 & 0.00 & 0.35 & -0.01 \\
56.4 & 0.00 & -0.59 & 0.01 \\
59.4 & -0.01 & 0.80 & -0.01 \\
59.3 & -0.16 & -0.09 & 0.00 \\
- MP structure & & & \\
\hline 17.5 & 0.00 & -0.39 & 0.00 \\
21.7 & 0.26 & 0.03 & 0.00 \\
48.9 & -0.10 & 0.07 & -0.03 \\
44.5 & 0.00 & -2.94 & 0.00 \\
45.8 & 0.00 & -2.84 & 0.00 \\
57.9 & 0.00 & -0.59 & 0.00 \\
59.0 & 0.00 & 0.49 & 0.00 \\
60.2 & -0.01 & -0.18 & 0.00 \\
59.7 & 0.10 & -0.01 & 0.00 \\
\hline \hline
\end{tabular}




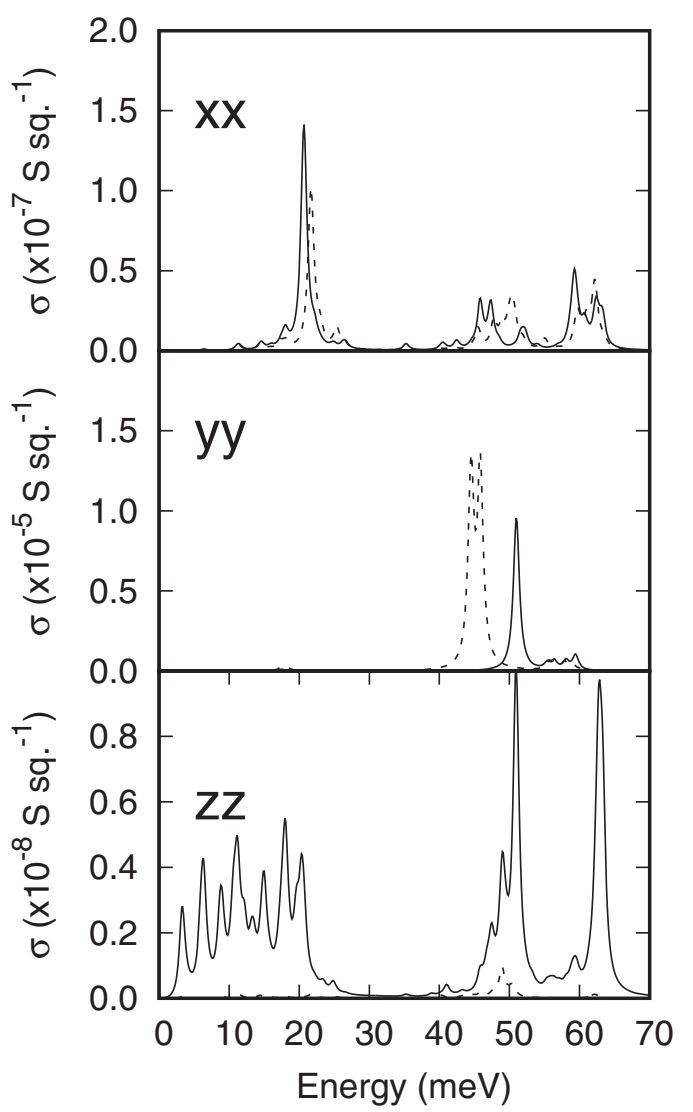

FIG. 3. Surface conductivity tensor elements for \pm MP surfaces in $\mathrm{S} \mathrm{sq}^{-1}+\mathrm{MP}$ (solid lines) $-\mathrm{MP}$ (dotted lines). Note differences in scales of axes. $x=[11 \overline{2}], y=[\overline{1} 10], z=[111]$.

a pair of modes polarized parallel to the chains at 44.5 and $45.8 \mathrm{meV}$ (previously $47.7 \mathrm{meV}$ ).
The authors are grateful to J. E. Demuth for pointing out the error in the original paper.
[1] M. Zitzlsperger, R. Honke, P. Pavone, and U. Schröder, Surf. Sci. 377-379, 108 (1997).

[2] G. Xu, B. Deng, Z. Yu, S. Y. Tong, M. A. Van Hove, F. Jona, and I. Zasada, Phys. Rev. B 70, 045307 (2004).

[3] G. Bussetti, B. Bonanni, S. Cirilli, A. Violante, M. Russo, C. Goletti, P. Chiaradia, O. Pulci, M. Palummo, R. Del Sole, P. Gargiani, M. G. Betti, C. Mariani, R. M. Feenstra, G. Meyer, and K. H. Rieder, Phys. Rev. Lett. 106, 067601 (2011).
[4] L. Reining and R. Del Sole, Phys. Rev. Lett. 67, 3816 (1991).

[5] J. E. Northrup, M. S. Hybertsen, and S. G. Louie, Phys. Rev. Lett. 66, 500 (1991).

[6] M. Rohlfing and S. G. Louie, Phys. Rev. Lett. 83, 856 (1999).

[7] C. Goletti, G. Bussetti, P. Chiaradia, and G. Chiarotti, J. Phys.: Condens. Matter 16, S4289 (2004). 